\title{
Téoros
}

Revue de recherche en tourisme

\section{Le tourisme en Abitibi-Témiscamingue...} La failble voix d'une région lointaine...

\section{Cécile Sabourin}

Volume 5, numéro 1, mars 1986

Tourisme et régions

URI : https://id.erudit.org/iderudit/1080584ar

DOI : https://doi.org/10.7202/1080584ar

Aller au sommaire du numéro

Éditeur(s)

Université du Québec à Montréal

ISSN

0712-8657 (imprimé)

1923-2705 (numérique)

Découvrir la revue

Citer cet article

Sabourin, C. (1986). Le tourisme en Abitibi-Témiscamingue... La faible voix d'une région lointaine... Téoros, 5(1), 5-40. https://doi.org/10.7202/1080584ar d'utilisation que vous pouvez consulter en ligne.

https://apropos.erudit.org/fr/usagers/politique-dutilisation/ 


\title{
La faible voix d'une région lointaine... Le tourisme en Abitibi-Témiscamingue...
}

\author{
par Cécile Sabourin *
}

Ni un historique du développement touristique en Abitibi-Témiscamingue, ni une analyse de l'éveil de la conscience de ce que représente l'industrie touristique pour une région comme l'Abitibi-Témiscamingue. cet article ne vise qu "à mettre en lumière le décalage entre les "apparences" et les véritables enjeux en ce qui concerne le développement d'une industrie touristique abitibienne et témiscamienne.

On parle de tourisme chaque fois que l'on se réfere à un déplacement de 24 heures ou plus, ayant pour objectif l'agrément, le travail, des raisons personnelles, etc. Phénomène restreint au début du siècle, le "tourisme" s"intègre maintenant à la vie de presque tout le monde et, parallèlement a la croissance de popularité du tourisme, une quantité importante de services se sont développés. Tout service foumi à la personne séjoumant à l'extérieur de son lieu habituel de résidence peut être considéré comme faisant partie intégrante de l'industrie touristique. Alors qu'il s'agit de débattre de l'industrie touristique comme facteur de développement, on constate l'ambiguité que suscite une telle définition. Comment distinguer les dépenses des résidents de celles des touristes? Comment identifier les items de consommation des tourises? Nombreuses sont le tentatives de mesurer l'ampleur des dépenses touristiques et leurs effets sur l'économie d'un territoiré, cependant l'évaluation de la place réelle du tourisme dans une société demeure difficile à réaliser. En Abitibi-Témiscamingue, il n'en est pas autrement.

Après avoir élaboré certains paramètres nous permettant de situer l'industrie touristique dans le contexte régional, nous évoquerons les contraintes d'une prise en mains du devenir touristique de la région par les intervenants concernés ainsi que les enjeux en cause.

Une région, deux visions... La perception officielle a rapidement classé I'Abitibi-Témiscamingue parmi les régions

"Cecile Sabourin est professeure a rUniversite du Qué bec en Abrtbi-Temiscamingue; elle a soutenu une these de doctorat a runiversite d'Aix-Marseille en 1885 sur e lounsme dans les regians rurales du Quebec: buar ot perspectives. ressources axées sur l'exploitation et l'expédition de ses ressources minières. forestières et énergétiques. Si l'on ajoute à cela l'ensemble des données touchant le phénomène de la colonisation, on possede un tableau à peu près complet de l'image véhiculée sur le passé, le présent et les perspectives d'avenir de la région. Les principaux acteurs et décideurs, intervenant sur la scène régionale, vouent un culte aux mesures économiques traditionnelles que sont le niveau et production et d'emploi par secteur industriel, le taux de chômage, l'investissement, les volumes de production, etc.

Ils se préoccupent peu des traits de caractère de la population, du milieu social et culturel et du vécu économique réel des habitants de la région. Certains s'inquiètent récemment du haut taux de suicide, de la stagnation de la population, des piètres services de santé, de la pollution, de la santé et sécurité au travail. Certains groupes s'interrogent et lancent des débats sur des enjeux qui les concernent. La réponse "institutionnelle" se fait sentir soit timidement, soit de manière directive en fonction des politiques centrales de l'État, c'est-à-dire en fonction des préoccupations véhiculées par et au bénéfice des élites politiques et economiques.

Les véritables facteurs générateurs de la santé économique, sociale et culturelle de la région que sont le climat social, les ressources humaines et leur capacité de se développer en harmonie avec leur environmement sont à peine pris en considération par les milieux institutionnels. Ils ne retiennent souvent l'attention que de groupes et d'individus dont les activités possedent une faible emprise sur les décisions de nature politique et economique. Sans viser à remettre en cause la vision généralement entretenue de ce qu'est une région dite éloignée des grands centres, ni la conception officielle des données socio-économiques pertinentes à l'analyse d'une société. ces remarques me permettent de situer dans un cadre plus global ma réflexion sur l'industrie touristique en Abitibi-Témiscamingue.

Du point de vue touristique, l'AbitibiTémiscamingue est, pour celle ou celui qui

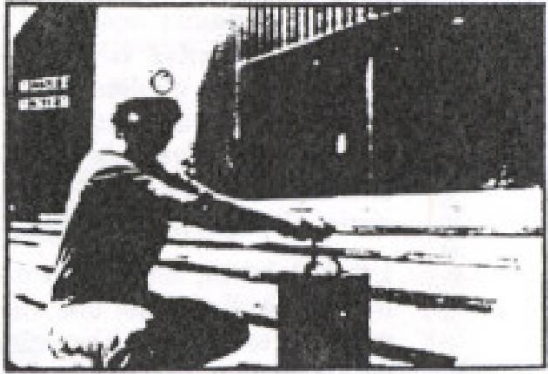

Le Musée minier de Malartic: un site touristi que qui reflete l'économie de la région.

connaît cette région à distance, un presque vide meublé uniquement de ressources de péche et de chasse, d immenses foréts, de sites de plein air. Et ils sont nombreux les Québécois et autres qui ne connaissent la région qu'à distance, quil sont peut-être venus ou viendront s'aventurer au-delà du parc de La Vérendrye pour quelques jours mais n'y découvriront comme prévu, en dehors de l'immensité de 'a nature, que quelques villes souvent accueillantes mais peu propices à les retenir. Inutile de se le cacher, presque tout est encore à faire pour créer en ce territoire de l'AbitibiTémiscamingue le "lieu" de tourisme que l'on publicise à l'extérieur de la région. On diffuse de la région une image qui correspond souvent à une réalite restreinte. S'il existe un véritable potentiel touristique susceptible d'entrainer des retombées intéressantes pour la région, il reste à identifier et a développer, pour que l'AbitibiTémiscaminque devienne le lieu de tourisme qu'elle aura choisi d'êtré

Le territoire régional est immense, bourré de charme et d'attraits lies aux grands espaces, à la beauté des sites sauvages, à la multiplicité des rivières et des lacs. Peu peupléc par une population qué l'on dit stagnante et vieillissante, I'Abitibi-Témiscamingue est une région neuve, ouverte depuis peu à force de sueurs québécoises et de capitaux étrangers. Elle vit au rythme de ses habitants qui n'ont pas eu le temps de se demander "pourquoi?" chaque fois qu "un législateur décidait d'une parcelle de leur présent et de leur avenir. Le vent de régionalisation pour ne pas dire la découverte de leur "régionalisme" a insufflé chez son élite un faible espoir de pouvoir penser et 
dire ses besoins et ses envies. Peu de secteurs y ont échappé. Comme la majorité des acteurs de la scène régionale, les intervenants touristiques ont cherché a acroitre leur influence sur l'avenir touristique régional sans toutefois se defaire de l'emprise exercée sur eux. Ils ont alors rêvé à l'image que la métropole avait d'eux et se sont atte: lés à la tăche d'y ressembler.

\section{Au delà des apparences}

L'image de l'Abitibi-Témiscamingue, on la retrouve dans les brochures publicitaires. D'envergure régionale, certaines brochures présentent la région à l'aide de photographies attrayantes et de commentaires qui feraient sursauter nombre $d$ 'amoureux de la vie abitibienne et témiscamienne. La documentation publiée inclut une liste très bien présentée des attraits régionaux ainsi que des brochures plus modestes sur les sites culturels et naturels et les circuits touristiques suggérés.

Les brochures, fournissant des renseignements spécifiques sur une ville, un site ou un attrait touristique, sont très souvent préparées avec les moyens limités que possède I'Association touristique régionale. On y décèle une grande préoccupation de recen. ser tous les attraits disponibles. Des brochures de conception plus élaborée sont disponibles soit pour la promotion de sites mieux organisés ou dans les cas où le financement est fourni par des organismes extérieurs et des commerçants participants.

Les résultats nous convainquent bien de l'effort déployé pour présenter et promouvoir le produit. Ils sont moins convaincants de l'atteinte d'un quelconque objectif qui pourrait se matérialiser par une industrie touristique florissante.

Il semble y avoir un ecart marqué entre ce pays neuf du point de vue touristique, doté de ressources intéressantes et commercialisées de façon peu originale et la conviction que l'industrie touristique d'Abitibi-Témiscamingue est économiquement et socialement rentable.

\section{Dans la foulée de la régionalisation}

Dans la foulée de la régionalisation des années "70 au Québec, le désir de prise en charge régional du devenir touristique de l'Abitibi-Témiscamingue n'a pas échappé à l'emprise des institutions. L'État québécois a favorisé, comme dans dix-sept (17) autres régions du Québec, l'émergence et l'organisation d'une association touristique régionale. Comme c'est le cas pour l'ensemble des associations et. conseils régionaux préoccupés de différents secteurs d'activités, les associations touristiques régionales (ATR) ont été constituées en vue de regrouper sous un mème chapeau les intervenants concernés par un domaine d'activités. Le rôle des ATR, selon le gouvernement québécois, est "de regrouper efficacement, sur une base géographique. les diverses energies touristiques de la région. En matièré de promotion, les $A T R$ oni la responsabilité de la mise en marché du produit régional et de l'accueil des visiteurs. En matière de développement elles jouent un role consultarif... "11' On ajoute que la mise sur pied de bureaux régionaux du ministère du Tourisme de la chasse et de la peche permettra "la coordination des effors consacrés à la préparation des schémas de développement touristique régionaux ${ }^{* 23}$ Depuis lors, le tourisme a acquis ses lettres de noblesse et atteint le rang d'une industrie au sein du ministère de l'Industrie, du Commerce et du Tourisme en 1979 puis a franchi, en 1984 , le stade ultime de la reconnaissance officielle lors de la création du ministère du Tourisme.

Comment la région de l'Abitibi. Témiscamingue a-t-elle vécu et continuet-elle de vivre l'émergence de ce nouvel appareil?

\section{Une ATR sous influence}

En l'absence de dêlégué au tourisme en Abitibi-Témiscamingue, l'Association touristique régionale de l'AbitibiTémiscamingue (ATRAT) devient l'interlocuteur principal du gouvernement québécois en ce qui concerne les questions touristiques et possède un rôle de premier plan, rôle qui lui confere une lourde tesponsabilité concernant les enjeux de I'industrie touristique régionale. Elle est officiellement un centre de regroupement des intervenants touristiques et un lieu de concertation de leur action.

Formée depuis 1978, l'ATRAT a déjà derrière elle une longue liste d'activités réalisées dans le cadre des objectifs qu'elle s'était fixés au départ. Elle a joué un rôle actif relativement à l'information, à l'accueil et à la promotion du tourisme en région. Elle s'est engagée dans des activités de développement du produit touristique et a effectué de multiples interventions aux niveaux provincial et régional. Enfin elle a participé à la mise sur pied de sessions de formation ainsi qu'à des projets spéciaux. L'énumération de ses activités ne réussit cependant pas à exprimer l'ampleur et la nature véritable du travail accompli.

On ne saurait en effet mesurer avec précision son travail de concertation des intervenants régionaux et sa responsabilité relativement à la concrétisation de projets qui touchent l'accueil, l'information et la promotion.

Si on le compare à celui du developpement, le volet "taccueil, information et promotion" constitue sans nul doute celui pour lequel l'ATRAT est le mieux préparée et le plus susceptible d'une action efficace. Parmi les raisons qui I'expliquent, notons l'importance des enjeux politiques concernés, la facilité de satisfaire des besoins d'accueil, d'information et de promotion dans le cadre de programmes de soutien temporaires, les plus faibles délais requis pour la mise en application d'activités, etc.
Ägée de 7 ans, l'ATRAT n'a pas encore atteint la maturité d'un intervenant en pleint possession de ses moyens au service d'objectifs bien identifiés. Elle demeure prisonnière de multiples contraintes issues de ses origines et de sa dependance par rapport à l'administration québécoise centrale. Occupee à considerer la réaction suscitế par ses activités aupres de ses membres el du ministere "subventionnaire" , elle $\pi$ " que peu de ressources à consacrer à l'identification et à l'analyse des véritables enjeux touristiques de la région. Faute de faire de vrais choix en fonction de la populatior d"ici, elle utilise ses énergies à développe: l'image que le ministère du Tourisme et se: propres membres ont d'elle et se débất pou susciter la participation et la concertatior des intervenants touristiques de la région

Au moment oủ le ministère du Tourisme s'apprète à mettre en place des moyens qui permettent aux régions touristiques de se doter de plans de développement, leu octroie-t-il parallèlement le choix d'orien ter leur développement en fonction d'objec tifs régionaux? Après avoir affirmé, lors du sommet économique sur le tourisme et dans les documents qui ont suivi, l'importance du tourisme comme industrie québécoise. les pouvoirs publics ont mis de l'avant de: orientations de développement faisan fi des régions avant un potentiel touristique pet affirmé. En identifiant les circuits, couloirs et póles de développement selon leur vocation internatinale ou nationale, on mettai en veilleuse une partie imporante des pro. blématiques régionales en matière de développement touristique, à savoir les besoins des résidents et les véritables retombées économiques et sociales du tourisme.

Dans l'exposé de la situation présentée la premiere page du Devis pour un plan de développement touristique de I'Abitibi-Témiscamingue, on insiste sur le róle de l'industrie touristique qui en $s e$ développant dans des régions moins indus. trialisées, contribue a anenuer les dispa rités economiques régionales ${ }^{3 i}$ Il $n^{\prime}$ 'es! alors fait aucune mention des conditions essentielles susceptibles d'assurer à une région comme l'Abitibi-Témiscamingue de véritables retombées positives d'une activité économique.

\section{S'il existait une autre voie?}

Le gouvernement québécois a affirmé sor option fondamentale d'axer le développe. ment du tourisme au Québec d'abord en fonction de ses finalités économiques. L'Association touristique régionale de l'Abitibi-Témiscaingue (I'ATRAT) et ses membres ne sont pas sans connaitre ces orientations globales du développement touristique québecois. S'ils en questionnen certaines, ils ne peuvent que se sentir peu impliqués dans la mise en place de ce "projet touristique" québécois choisi et affirme par l'État dans l'ensemble de ses énoncés de politique.

(Suite of ha page 4O) 
DAVIS, K. Pascal and Bernard W. TAYLOR, $A_{4}$ goal programming model for allocating state promotional effort to regional markers in accordance with tourism potentiel, Journal of Travel Pesearch, vol, XVIII, no 4 (Spring 1900), pp. 24-30.

EDGELL, David and Richard SEELY, A multistage model for the development of interna. tional rourism forecasts for States and Regrons, in Tourism Planning and Development lissues, Washington, D.C.: George Washington University, 1980 , pp. 407-410.

GUNN, Clare A., An approach to regional assessment of tourism development potential, in Tourism Planning and Development lssues, Washington, D.C.; George Washington University, $1980, \mathrm{Pp}, 261-276$

GUNN, Clare A., Vacationscape: designing tourist regions. Austin (Texas): University of Texas, $1972,238 \mathrm{p}$
NiCHOLLS, Leland, L., Regional Tourism deve lopment in "Third World Americaw: a propo sed model for Appalachia, in Tourism Planning and Development issuess, Washington, D.C. George Washington University, 1980, pp 283-294

PALMER, John and Gordon PROBERT, Planning at the county level: a case study of Gwent, South Wales, Tourism Management. vol. 1, no 3, isept. 1980), pp. 158-167.

RITCHIE, J.R. Brent and Michel ZINS, Culture as determinant of the attractiveness of a tou rism region, Annals of Tourism Research, vol. $\checkmark$ no 2 (april/june 1978), pp. 252-267.

\section{Population et intéréts régionaux}

KNOPP, Timothy B., Tourism, the local interests and the function of public lands, in Tou-

rism Planning and Development Issues. Washington, D.C.: George Washington University, 1980, pp, 225238.

LOUKISSAS, P.J., Public participation in com munity tounism planning a gaming simulation approach, Journal of Travel Research, wol. XXII, ho 1 (summer 1983). pp. 18-23.

MOULiN, Claude, Plan for ecological and culfural rourism involwing particioation of loca! population and associations, in Tourism Planning and Development issues. Washington. D.C: George Washington University, 1990 , pp. 199-212.

PIZAM, Abraharn and Ernest J. ACQUARD, Some social costs and nebefits of tourism to rural communities: the Cape Cod case. Amherst (Mass. I: Massachusetts Agricultural Experiment Station, 1977, 84 p. $f$

gue refont les gestes attendus d'eux et calqués sur les modèles sans au préalable tenter de se définir et de se développer en fonction des besoins de leur région et de leur population

L'Abitibi-Témiscamingue a une spécificité touristique qui requiert, pour être exploitée de façon rentable au profit des résidents, qu'on s'y attarde et qu'on la développe. Dans ce pays neuf, depuis peu éveillé à l'idée que le tourisme peut y trouver place. la tentation est forte et le danger est grand de chercher à ressembler aux autres et de développer un produit touristique sur les mémes bases que les autres régions, en utilisant leurs paramètres, en suivant leur modèle.
L'Abitibi-Témiscamingue porte le poids du déséquilibre entre les grands centres et la périphérie, entre les grands enjeux économiques et le bien-être de la population, entre le tourisme générateur de retombées économiques et le tourisme générateur de santé sociale. Loin de choisir et de se situer face à ces grands enjeux, elle se met en file et quête sa part du gâteau. Et si l'avenir passait par une autre voie... $f$

\section{Adeferences}

(1) Gouvernement du Quebec, Batir le Qubbec. Enonce de politique economique, 1979, p. 411 .

(2) Idem, p. 412

(3) Document interne de la Direction de Fanalvse et du developpement. Ministere du Tourisme, 3 juin 1985.
(Suite de la page 35)

\section{Le développement et I'aménagement touristiques}

touristique régionale du Pays-de-L'Érable lors de l'élaboration du plan de développement touristiqué régional et sont actuellement pris en compte dans les schémas d'aménagement des MRC concernées.

À l'heure de la réalisation des projets reliês au couloir Beauce-Appalaches, peut-on espérer que le contrat de développement sera la formule idéale pour soutenir le développement touristique? Nous serons sûrement en mesure d'ici quelques mois de vérifier si l'objectif de cette formule a èté rempli. Ainsi, suite à l'expérimentation de la formule des accords-cadres de développement, 1'OPDQ devra remettre un rappor d'évaluation au gouvernement avant l'élaboration d'accords-cadres additionnels.

La Loi sur l'aménagement et l'urbanisme aura donc permis de susciter une nouvelle mentalité ouverte à la planification et à la concertation non pas seulement du milieu municipal mais aussi de plusieurs autres intervenants socio-économiques.

L'expérience des plans de développement touristique, pour leur part, nous démontre l'intérêt đu milieu municipal face à la planification de développement touristique régional par la participation de leur(s) représentant(s) au Comité de gestion.

Les dix contrats de développement qui seront préparés prochainement nous permettent de connaître davantage le degré de volonté des $\mathrm{MRC}$ de s'impliquer dans le développement régional. Par ailleurs, les deux (2) contrats de développement touchant, entre autres, le tourisme permettront de constater si la cohésion entre les intervenants politiques et socio-économiques est possible

La planification tant du développement tou ristique que de l'aménagement est donc en devenir; l'étal actuel de la situation nous permet d'être optimiste quant à la poursuite du processus.

Par contre, après l'etape de la planification, on devra passer à la mise en application des plans et schémas. Cette cohésion, qui a été amorcée depuis, peu, se poursuivra-t-elle? $\mathrm{L} a$ volonté de concertation et de conciliation des intervenants sera à ce moment déterminante $f$

\section{Rofbrences}

(11) Gouvernement du Quebec: Batir le Qubbec. Enance de politique "economique, 1979 Pp. $411-412$.

(2) Idem

(3) Gouvemement du Quebec: Le tourisme au Que. bec. 1984, р. 9 .

(4) GRAVEL. Robert, La crbation des MRC et la dbcentralisation gouvernementale: analyse et dvaluation, ENAP, 1983, p. 33

(5) Gouvernement du Guebec: Le choin des regions. 1983. D. 80 .

(6) OHfice de planification et de developpement du Quebec: Le contrat de devaloppement, 1985. p. 1. idocument peesente lors du Congress de I'Union des municipalites regionales de cornte et des municipalités locales du Quebee? 